\title{
Perfil fisiológico de biomarcadores séricos durante uma prova com ciclistas de elite
}

\author{
Physiological profile of serum biomarcadores during a proof with elite cyclists
}

Jaqueline Santos Silva Lopes ${ }^{1,2 *}$ jaqueee-santosss@hotmail.com

Claudia Marlise Balbinotti Andrade ${ }^{1}$ claudia.mb.andrade@hotmail.com

Rosângela Ferreira Lustosa ${ }^{2}$ rosangela_lustosa@hotmail.com

Aníbal Monteiro Magalhães Neto ${ }^{3}$ professoranibal@yahoo.com.br

Projeto de pesquisa aprovado pelo Comitê de Ética em Pesquisa envolvendo seres humanos da Universidade Federal de Mato Grosso, sob o número: 1.064 .808 .

*Autor Correspondente

Jaqueline Santos Silva Lopes. R. Moreira Cabral, $n^{\circ} 1000$ - Barra do Garças/ Mato Grosso, Brasil. CEP: 78600-000

Telefone: +55 66999202206

Endereço científico: Universidade Federal de Mato Grosso (UFMT)

${ }^{1}$ Univ. Federal de Mato Grosso, Programa de Pós Graduação em Ciências da Saúde, Cuiabá, Brazil.

${ }^{2}$ Centro Universitário do Vale do Araguaia, Barra do Garças, Brazil. ${ }^{3}$ Univ. Federal de Mato Grosso, campus do Araguaia, Barra do Garças, Brazil.

\begin{abstract}
Resumo
Introdução: Verifica-se lacuna referente a investigações de parâmetros fisiológicos em modelo esportômico, responsável por caracterizar cenário real em ciclistas.

Objetivo: Investigar o comportamento fisiológico, por meio de análises da glicose, taxa hormonal e dano muscular nos momentos pré e pós competição de mountain bike, do tipo $\mathrm{XCO}$.

Métodos: Dez ciclistas de elite (média \pm DP idade $23 \pm 2,3$ anos, altura $175 \pm 7,8 \mathrm{~cm}$, massa corporal $68,5 \pm 6,1 \mathrm{~kg}$ ) foram incluídos no estudo. As coletas de dados foram realizadas por meio de análise sanguínea, verificadas nos momentos 24 horas antes (M1), imediatamente após (M2), 1 (M3) e 24 horas (M4) após a prova.

Resultados: Em geral, houve aumento significativo de todos os parâmetros analisados entre M1 e M2. A testosterona e a insulina demonstraram pico em M2 e M3, respectivamente $(96,15 \mathrm{ng} / \mathrm{Dl} ; 6,52 \mathrm{UI} / \mathrm{ml})$. Para o cortisol verificou-se pico em M2 (32,06 $\mathrm{ug} / \mathrm{dl})$, que apresentou diferença significativa $(\mathrm{p}=0,0001)$. O lactato, demostrou diferença estatisticamente significante $(\mathrm{p}=0,0002)$ entre M1 e M2. Para glicose, houve valor de pico em M2 $(105,4 \mathrm{mg} / \mathrm{dL})$, com diferença estatisticamente significante $(\mathrm{p}=0,0110)$. Em M4 todas as concentrações retornaram aos valores basais.

Conclusão: Os desfechos possuem potencial intrínseco para fundamentar a prática relacionada com as respostas metabólicas verificadas, permitindo aplicações de ações específicas que visem otimizar as condições físicas do atleta.
\end{abstract}

Palavras Chave: Ácido Láctico. Medicina Esportiva. Fisioterapia. Ciclismo. Ciclismo de Estrada. Glicemia.

\begin{abstract}
Summary
Introduction: There is a gap regarding investigations of physiological parameters in a spore model, responsible for characterizing the real scenario on cyclists.

Objetive: The objective of this study was to investigate the physiological behavior, by means of glucose, hormonal rate and muscle damage analyzes in the pre and post competition moments of mountain biking, type XCO.

Methods: Ten elite cyclists (mean \pm SD age $23 \pm 2.3$ years, height $175 \pm 7.8 \mathrm{~cm}$, body mass $68.5 \pm 6.1 \mathrm{~kg}$ ) were included in the study. Data collection was performed by means of blood analysis, verified at 24 hours before (M1), immediately after (M2), 1 (M3) and 24 hours (M4) after the test.

Results: In general, all parameters analyzed between M1 and M2 were increased significant. Testosterone and insulin demonstrated a peak in M2 and M3, respectively (96.15ng / dl, $6.52 \mathrm{IU} / \mathrm{ml}$ ). For cortisol, peak was observed in M2 (32.06 ug / dl), which presented a significant difference $(\mathrm{p}=0.0001)$. Lactate showed a statistically significant difference $(\mathrm{p}=0.0002)$ between M1 and M2. For glucose, there was a peak value in M2 (105.4mg / dL), with a statistically significant difference $(\mathrm{p}=0.0110)$. In M4 all concentrations returned to baseline.

Conclusion: The outcomes have intrinsic potential to support the practice related to the verified metabolic responses, allowing applications of specific actions aimed at optimizing the athlete's physical conditions.
\end{abstract}

Keywords: Lactic Acid. Sports Medicine. Physical Therapy Specialty. Bicycling. Road Cycling. Blood Glucose.

\section{Cite como \\ Vancouver}

Lopes, JSS, Andrade CMB, Lustosa, RF, Magalhães Neto, AM. Perfil fisiológico de biomarcadores séricos durante uma prova com ciclistas de elite. Conscientiae Saúde 2019 out./dez.;18(4):470-479. https://doi.org/10.5585/conssaude.v18n4.15381. 


\section{Introdução}

O mountain bike (MTB) constitui modalidade esportiva praticada em cenários naturais e com diversidades climáticas, nos quais o praticante passa por obstáculos com terrenos escorregadios, acidentados e irregulares, fatores que exigem destreza, controle emocional, superação de limites e condicionamento físico adequado ${ }^{1}$.

Esta modalidade esportiva é dividida em três tipos sendo eles, cross-country (XCO), montanha maratona de bicicleta (XCM) e o downhill (DHI). Desses, o modelo XCO é caracterizado por percurso extenso de longa duração ${ }^{2}$. Além disso, as características inconstantes do percurso, constituído porterrenos acidentados, com obstáculos, torna necessário que o ciclista recrute diferentes vias metabólicas, para síntese de energia. Dessas, as vias fosfato de alta energia e glicolítica lática, desempenham papel predominantes, sendo recrutadas durante os momentos de subida, para passar um obstáculo e em momentos onde são necessários explosão ${ }^{3}$.

Neste cenário, estudos apontam que os estresses mecânico e metabólico são considerados fatores iniciais relacionados a ocorrência de dano muscular induzido pelo exercício, especificamente nesta modalidade esportiva. As alterações fisiológicas podem ainda, ocasionar mudanças na estrutura do sistema nervoso, endócrino e imune $\mathrm{e}^{3,4}$.

A exemplo, outros estudos afirmam que, em condições de estresse metabólico exacerbado, o sistema imunológico pode ser afetado de maneira significativa, aumentando condições para o desenvolvimento de doenças infecciosas ${ }^{5,6}$. Também, durante a prática esportiva ocorre aumento da permeabilidade do sarcolema, com acúmulo contínuo de lactato na corrente sanguínea, responsável por causar dor muscular tardia. Outro parâmetro importante, se refere as taxas hormonais, que quando desequilibradas, tendem a propiciar fadiga e dor antecipadas, que prejudicam o desempenho subsequente ${ }^{7-9}$.

Fatores extrínsecos relacionados ao tipo de exercício físico, duração, intensidade e condição física, associados às condições ambientais, determinam níveis de alterações da homeostase fisiológica basal ${ }^{6-8}$. Neste sentido, é importante entender as respostas desencadeadas por modalidades esportivas específicas, tendo em vista que cada esporte possui suas características particulares.

Hipotetiza-se que os desfechos encontrados demonstrarão elevação das concentrações hormonais analisadas, em decorrência do tipo de estresse extenuante provocado durante a realização da modalidade esportiva investigada. Assim, a partir do exposto, levando-se em consideração as exigências fisiológicas demandadas durante a competição de XCO, parece 
pertinente a implementação de investigações que verifiquem os parâmetros descritos em resposta a prática dessa modalidade esportiva, para servir de parâmetro em estudos futuros que considerem dinâmicas de prescrição de treinamento e prescrição de técnicas recuperativas, especificas para as necessidades individuais verificadas, relacionadas à possível condição de sobrecarga ou recuperação inadequada. Portanto, este estudo teve como objetivo investigar as taxas de variações hormonais (insulina, cortisol e testosterona), lactato e glicose nos momentos subsequentes a uma prova de mountain bike no formato XCO.

\section{Métodos}

\section{Participantes do estudo}

A amostra foi composta por dez atletas, do sexo masculino (idade: $23 \pm 2,3$ anos; estatura: $175 \pm 7,8 \mathrm{~cm}$ e massa corporal: $68,5 \pm 6,1 \mathrm{~kg}$ ), praticantes da categoria elite regional de mountain bike, inscritos na Federação de ciclismo do estado de Mato Grosso (Brazil). Não foram incluídos participantes que apresentassem as seguintes características: ser etilista, consumir drogas, fumo ou medicamentos anti-inflamatórios de forma crônica, apresentar anemia, processo inflamatório, diabetes, doença cardiovascular e episódio de lesão músculotendínea ou osteoarticular nos membros inferiores e/ou coluna nos últimos seis meses. Além disso, seriam excluídos participantes que por quaisquer razões não aceitassem participar ou não completassem todas as etapas do estudo ${ }^{9}$. Entretanto, não foram registradas perdas de seguimento de modo que, todos os participantes incluídos inicialmente tiveram seus dados analisados.

\section{Declaração de ética}

Todos os participantes foram informados sobre os objetivos e procedimentos do estudo e, após concordarem assinaram um termo de consentimento livre e esclarecido (TCLE), assegurando sua privacidade. O estudo foi aprovado pelo comitê de ética de pesquisa com seres humanos da Universidade Federal do Mato Grosso, campus de Araguaia sob parecer: 1.064.808.

\section{Delineamento do Estudo}

Trata-se de um estudo com delineamento observacional. $\mathrm{O}$ estudo foi realizado durante uma etapa do Campeonato Mato-Grossense de Mountain Bike/2012, realizado na cidade de 
Barra do Garças (MT) com base no modelo de competição de Cross Country Olímpico (XCO). Os participantes foram instruídos a não realizar exercícios físicos vigorosos no período de 24 horas anterior ao início da prova e realizar refeição leve, habitual.

A coleta de dados foi caracterizada por análise sanguínea dos participantes nos momentos basal (M1), imediatamente após o esforço (M2), após 1 hora (M3) e após 24 horas (M4)da prova. Os seguintes desfechos foram analisados: insulina, testosterona, cortisol, lactato e glicose.

Houve mascaramento dos participantes e responsável pela coleta sanguínea que não sabiam quais análises seriam realizadas ${ }^{10}$. Além disso, o responsável pelas analises, não tinha conhecimento sobre o esporte ou momentos de coletas.

\section{Procedimentos}

\section{Protocolo de exercício - XCO}

A prova de XCO foi realizada em circuito e constituído por um número definido de voltas, onde todos os participantes partiram juntos em um único pelotão. A pista de competição possuía a extensão de $2,4 \mathrm{~km}$, sendo a prova constituída por um total de 20 voltas. O protocolo utilizado seguiu em consonância com as normas do esporte, descrito em estudo anterior ${ }^{11}$.

\section{Características do percurso}

O percurso apresentou elevação na partida, caracterizando subida, em relação a chegada de 25 metros em terreno acidentado. A prova foi realizada em um sábado, com início às 9:00 horas da manhã. A temperatura registrada no dia foi de $28{ }^{\circ} \mathrm{C}$ com $60 \%$ umidade relativa do ar. A velocidade média por volta dos atletas foi equivalente a $23,2 \mathrm{~km} / \mathrm{h}$.

\section{Coleta de dados e análise sanguínea}

O método de coleta foi baseado no modelo Esportômico ${ }^{11}$, caracterizado por realizar a coleta de sangue no ambiente real de competição do atleta. Para tanto, foram coletados $10 \mathrm{ml}$ de sangue da veia antecubital do braço direito, posteriormente armazenados em tubos para coleta de sangue a vácuo, de vidro, com solução aquosa de etilenodiaminotetracetado dissódico a 10\% (EDTA). As amostras de sangue foram ordenadas da seguinte maneira: momento 1 (24 horas antes da prova - M1),momento 2 (imediatamente após a competição - M2),momento 3 (1 hora depois da prova - M3) emomento 4 (24 horas após o término do percurso - M4). As 
coletas de material sanguíneo foram realizadas por profissionais de enfermagem e biomedicina com expertise em realizar coletas de campo.

O sangue foi centrifugado a $2500 \mathrm{rpm}$ em uma centrífuga clínica durante 10 minutos, sendo o soro obtido usado imediatamente para a determinação quantitativa dos níveis séricos da enzima lactato. Utilizou-se kits comerciais Bioclin ${ }^{\circledR}$, que catalisa a oxidação do L-Lactato a Piruvato, com consequente redução do NAD+ a NADH. A concentração de L-Lactato é medida pelo aumento da absorbância a $340 \mathrm{~nm}$, a partir da formação de NADH. Para dosar a glicose, utilizou-se ensaio imunoenzimático (kit ELISA para glicose da R \& D Systems). Tratase de uma ferramenta confiável e validada, baseada na utilização de anticorpos para detectar e quantificar antígenos de interesse. Os hormônios (testosterona, insulina e cortisol) foram dosados por meio do kit Enzo Life Sciences, Inc também, reportado em estudos anteriores.

\section{Análise Estatística}

Para todas as variáveis investigadas, foram calculados valores de média e desvio padrão. A condução da análise estatística foi realizada por meio do pacote software SPSS 2.0.

A significância estatística entre grupos foi calculada utilizando a análise do teste t. As diferenças significativas foram estabelecidas em $\mathrm{P}<0,05(5 \%)$.

\section{Resultados}

Os resultados verificados, relacionados ao comportamento fisiológico nos momentos prévio e subsequentes à uma prova de ciclismo são apresentadas na tabela 1 e figura 1 , e referem-se as análises sobre marcador bioquímico (lactato), glicose e dosagem hormonal (cortisol, insulina e testosterona). Em geral, houve aumento de todos os parâmetros analisados entre os momentos 24 horas antes do esforço e imediatamente após o esforço, com valores de pico no momento imediato após o esforço, exceto para insulina, que apresentou pico em M3.

No que se refere a taxa hormonal, as análises da testosterona demonstraram pico no momento após a competição (96,15ng/D1), com declínio gradual nos momentos subsequentes (1 h e 24 hrs após a competição). Os desfechos visualizados sobre o comportamento da insulina, demonstraram valor de pico no momento de 1 hora após a competição $(6,52 \mathrm{UI} / \mathrm{ml})$ porém, não foram observadas diferenças significativas, entre os momentos $(\mathrm{p}<0,05)$. Os valores de cortisol, demonstraram valor de pico acentuado após a competição $(32,06 \mathrm{ug} / \mathrm{dl})$, que apresentou diferença significativa entre os demais momentos analisados $(\mathrm{p}=0,0001)$. 
As análises referentes ao marcador bioquímico (lactato), mostraram diferença estatisticamente significante ( $\mathrm{p}=0,0002)$ entre os momentos $24 \mathrm{~h}$ antes e imediatamente após a competição.

Para valores da glicose, verificou-se pico no momento após a competição (105,4mg/dL), com diferença estatisticamente significante entre os demais momentos avaliados $\left(\mathrm{p}=0,0110^{*}\right)$.

Tabela 1 - Valores de média e DP do marcador bioquímico de danos ao muscular (lactato), dosagem hormonal (cortisol, testosterona e insulina) e glicose nos momentos $24 \mathrm{~h}$ pré-exercício, imediatamente após, 1 h e 24 h após a competição

\begin{tabular}{|c|c|c|c|c|c|}
\hline & $24 \mathrm{~h}$ antes & $\begin{array}{c}\text { Após } \\
\text { competição }\end{array}$ & $1 \mathrm{~h}$ & $24 \mathrm{~h}$ & $p$-valor \\
\hline \multicolumn{6}{|l|}{ Hormônios } \\
\hline $\begin{array}{l}\text { Testosterona } \\
\text { livre }(\mathrm{ng} / \mathrm{dL})\end{array}$ & $83,32 \pm 26,9$ & $96,15 \pm 39,54$ & $87,05 \pm 27,9$ & $85,06 \pm 29,7$ & 0,1905 \\
\hline Insulina UI/ml & $2,71 \pm 2,2$ & $2,89 \pm 2,1$ & $6,52 \pm 7,3$ & $2,48 \pm 1,5$ & 0,5600 \\
\hline Cortisol ng/dL) & $13,79 \pm 3,3$ & $32,06 \pm 4,0$ & $22,37 \pm 6,30$ & $13,66 \pm 3,54$ & $0,0001^{*}$ \\
\hline \multicolumn{6}{|l|}{$\begin{array}{l}\text { Marcador } \\
\text { bioquímico }\end{array}$} \\
\hline $\begin{array}{l}\text { Lactato } \\
\text { (mg/dL) }\end{array}$ & $25,9 \pm 10,7$ & $92,9 \pm 35,6$ & - & - & $0,0002 *$ \\
\hline \multicolumn{6}{|l|}{ Carboidrato } \\
\hline Glicose (mg/dL) & $94,2 \pm 7,9$ & $105,4 \pm 38,6$ & $83,5 \pm 4,6$ & $74,1 \pm 7,1$ & $0,0110 *$ \\
\hline
\end{tabular}

\section{Discussão}

No presente estudo, objetivou-se verificar a variação hormonal, glicose e lactato nos momentos pré e pós competição de mountain bike no formato Cross country olímpico (XCO). Os principais achados dessa investigação referem-se as respostas metabólicas aumentadas desencadeadas em resposta a prática de mountain bike em atletas profissionais. Em geral, valores de pico foram verificados após o esforço, com redução graduação nos momentos subsequentes analisados.

O organismo realiza ajustes constantes para regular mudanças necessárias para manutenção da prática esportiva. A este respeito, estudos anteriores ${ }^{12-16}$ verificaram que os hormônios desempenham papel regulador na fisiologia, influenciando reações e funções na parte tecidual biológica. Dessa forma, o exercício físico exige mudanças na fisiologia do corpo, 
que causa alterações hormonais, tanto na testosterona como no cortisol ${ }^{17,18}$, verificados neste estudo.

A este respeito, a literatura relaciona valores de testosterona e cortisol com condições de equilíbrio anabólico e catabólico. Desse modo, tais hormônios, têm sido utilizados como biomarcadores para diagnóstico de overtraining e sobrecarga, ao fornecer estimativa sobre a intensidade do esforço praticado bem como, níveis de recuperação. Ainda, os dados observados, demonstraram recuperação dos níveis hormonais, no momento de 24 horas. Tal dado, sugere que o nível de treinamento dos participantes, não se enquadra, aparentemente em cenário de sobrecarga.

Ainda, no que se refere as taxas hormonais, a análise de cortisol demonstrou níveis elevados no momento imediato ao término da prova. Neste sentido, estudos ${ }^{19,20}$ demostraram que esse hormônio está ligado a degeneração de proteínas, em decorrência a uma resposta aguda ao treino de força, responsável por aumento significativo em indivíduos treinados. Esse aumento de cortisol, relaciona-se ao estresse oxidativo e dano muscular, causados pelo exercício. Esse estudo, demonstra ainda, que o nível do cortisol pode se manter elevado por vários dias, em detrimento da intensidade do esforço aplicado. No entanto, os desfechos verificados neste estudo, demonstram recuperação total no momento 24 horas após o esforço, o que novamente, demonstra que o esporte investigado, não parece causar níveis extenuantes de fadiga e sobrecarga, especificamente no perfil atlético investigado.

Com relação aos níveis de lactato, estudos anteriores ${ }^{21-24}$ demostraram que o exercício físico de alta intensidade é responsável por acúmulo de ácido lático no tecido muscular. Entretanto, atletas com uma boa condição física conseguem reduzir o lactato nos músculos durante o esforço físico. Neste estudo, os resultados mostraram um aumento significativo na concentração de lactato nos momentos subsequentes ao esforço, quando comparados aos valores coletados em repouso, o que condiz com situação fisiológica bem compreendida e esperada, após realização de atividades que exigem esforço extenuante.

No que se refere aos valores glicêmicos, estudos demonstram diminuição diretamente relacionada à intensidade e volumes exigidos no esporte, e ainda, relação inversamente proporcional aos níveis de cortisol ${ }^{25-27}$. Em concordância, os desfechos encontrados neste estudo, também reportaram aumentos de cortisol após a competição e redução nos níveis de glicemia.

Os pontos fortes desse estudo incluem a realização das coletas e procedimentos em ambiente de campo, que corresponde ao cenário real de competição utilizado na prática esportiva. Além disso, foram incluídos atletas de alto rendimento. Desse modo, os desfechos 
refletem respostas metabólicas fidedignas em resposta a prática de MTB, na modalidade XCO. Por outro lado, seria importante que novas pesquisas relacionadas às análises fisiológicas, de variáveis não investigadas neste estudo como por exemplo citocinas e creatina quinase.

Finalmente, no que diz respeito as implicações práticas, acreditamos que os desfechos apresentados fornecem parâmetros clínicos sobre o estudo das respostas fisiológicas desencadeadas em resposta à prática do ciclismo, em atletas de elite. Tais dados podem ser importantes na dinâmica de prescrição da periodização, auxiliando no desempenho esportivo e minimizando condições sobrecarga. Além disso, os dados apresentados também constituem potencial intrínseco para guiar a proposição de protocolos de recuperação após o esforço, na população estudada.

\section{Conclusão}

A partir dos dados apresentados, pode-se concluir que uma prova de XCO foi responsável por desencadear alterações metabólicas agudas, indicando nível de estresse em resposta à esse tipo de esforço, o que no entanto, não parecem perdurar pelas horas subsequentes. Os desfechos do presente estudo demonstram potencial para ser utilizado como parâmetro para identificar os limiares de mudança metabólica nestes atletas, auxiliando ainda pesquisas futuras. Ainda, o acompanhamento das variações hormonais pode auxiliar na prescrição de uma recuperação muscular mais adequada, enquanto o acompanhamento do Lactato apresenta utilidade no estudo da resposta do músculo ao treinamento, permitindo obter maiores indicações sobre a adaptação metabólica ao exercício físico.

Acredita-se que os dados apresentados, fortalecem as bases teóricas que fundamentam ciência e prática relacionadas ao treinamento físico e fisioterapia desportiva permitindo, aplicações de ações específicas que visem otimizar as condições físicas do atleta de MTB, em diferentes momentos.

\section{Conflitos de Interesse}

Os autores declaram não conhecerem conflitos de interesse relacionados ao manuscrito.

\section{Referências}

1. Peinado AB, Romero-Parra N, Rojo-Tirado MA, Cupeiro R, Butragueño J, Castro EA, et al. Physiological Profile of an Uphill Time Trial in Elite Cyclists. INT J SPORT PHYSIOL. 2018; 13(3):268-273. doi:10.1123/ijspp.2016-0768. 
2. Santos FJ, Santos AGC, Morais KA, Mendes CRS, Oliveira VM, et al. Análise do Perfil dos Praticantes de Mountain Bike (MTB) da Cidade de Trindade (GO). Rev da Faculdade União Goyazes 2016; 10(1):22.

3. Barber JJ, McDermott AY, McGaughey KJ, Olmstead JD, Hagobian TA. Effects of combined creatine and sodium bicarbonate supplementation on repeated sprint performance in trained men. J Strength Cond Res 2013; 27(1): 252-258.

4. Martínez AC, Pons MM, Gomila AS, Marí JAT, Biescas AP, Et al. Changes in circulating cytokines and markers of muscle damage in elite cyclists during a multi-stage competition. Clin Physiol Funct Imaging. 2015;35(5):351-8.

5. Simpson RJ, Kunz H, Agha N, Graff R. Exercise and the Regulation of Immune Functions. Prog Mol Biol Transl Sci. 2015;135:355-80. doi: 10.1016/bs.pmbts.2015.08.001.

6. Walsh NP. Recommendations to maintain immune health in athletes. Eur J Sport Sci. 2018;18(6):820-831. doi: 10.1080/17461391.2018.1449895.

7. Hayes LD, Grace FM, Baker JS. et al. Exercise-Induced Responses in Salivary Testosterone, Cortisol, and Their Ratios in Men: A Meta-Analysis Sports Med 2015; 45: 713. https://doi.org/10.1007/s40279-015-0306-y.

8. Lopes JSS, de Magalhães Neto AM, Alves PRL, et al. Kinetics of Muscle Damage Biomarkers at Moments Subsequent to a Fight in Brazilian Jiu-Jitsu Practice by Disabled Athletes. Front. Physiol. 2019; 10: 1055.

9. Lopes JSS, Micheletti JK, Machado AF, et al. Test-retest reliability of knee extensors endurance test with elastic resistance. PLoS One 2018; 13 (8): e0203259.

10. Lopes JSS, Santos SP, Almeida LMB, et al. Efficacy of Ultrasound for Localized Fat Treatment on Clinical and Psychological Outcomes: A Randomized, Single-Blind, Placebo-Controlled Clinical Trial. Aest Surg J Open Forum 2020;ojaa012. Doi: https://doi.org/10.1093/asjof/ojaa012.

11. Araújo NC, França AM, Cameron LC, Magalhães Neto AM. Análise de biomarcadores de soro de lesão do músculo durante Competição bicicleta de montanha. Conscientiae Saúde 2016, 15.

12. Bassini A, Cameron LC. Sportomics: building a new concept in metabolic studies and exercise science. Biochem Biophys Res Commun. 2014;445(4):708-16.

13. Abbasi A, Fehrenbach E, Hauth M, Walter M, Hudemann J, Wank V, et al. Changes in spontaneous and LPS-induced ex vivo cytokine production and mRNA expression in male and female athletes following prolonged exhaustive exercise. Exerc. Immunol. Rev. 2013; 19: 8-28.

14. Muhamad AS, Kiew Ooi F, Meor-Osman J, Chen CK. The effects of combined probiotic ingestion and circuit training on muscular strength and power and cytokine responses in young males. APPL PHYSIOL NUTR ME. 2018, 43(2): 180-186, https://doi.org/10.1139/apnm-2017-0464

15. Mauer J, Chaurasia B, Goldau J, MC Vogt, Ruud J, Nguyen KD, et al. IL-6 signaling promotes the alternative activation of macrophages to limit endotoxemia and insulin resistance associated with insulin. Nat. Immunol. 2014; 15 (5): 423-430.

16. Shoelson SE, Lee J, Goldfine AB. Inflammation and insulin resistance. J. Clin. Investir. 2006; 116 (7): 1793-1801.

17. Resende NM, de Magalhães Neto AM, Bachini F, de Castro LE, Bassini A, Cameron LC. Metabolic changes during a field experiment in a world-class windsurfing athlete: a trial with multivariate analyses. OMICS. 2011;15(10):695-704.

18. França SC, Barros Neto TL, Agresta MC, Lotufo RF, Kater CE. Resposta divergente da testosterona e do cortisol séricos em atletas masculinos após uma corrida de maratona. Arq Bras de Endocrin \& Metabol. 2006;50(6):1082-1087. 
19. Paunksnis MR, Evangelista AL, La Scala Teixeira CV, Alegretti JG, Pitta RM, Alonso AC, Figueira A Jr, Serra AJ, Baker JS, et al. Metabolic and hormonal responses to different resistance training systems in elderly men. Aging Male. 2018;21(2):106-110. doi: 10.1080/13685538.2017.1379489.

20. Simonetto L, Fiorella P, Impellizzeri FM, et al. Testosterone and cortisol in 93 elite road cyclists during a 10-day stage race: relationship with final ranking. Sport Sci Health. 2016; 12: 407. https://doi.org/10.1007/s11332-016-0306-9.

21. Karasiak FC, Guglielmo LGA. Effects of Exercise-Induced Muscle Damage in WellTrained Cyclists' Aerobic and Anaerobic Performances. J Strength Cond Res. 2018; 32(9): 2623-2631. doi: 10.1519/JSC.0000000000002522.

22. Engel FA, Härtel S, Sperlich B, Holmberg AC. The kinetics of blood lactate in boys during and following a single and repeated all-out sprints of cycling are different than in men - Do children indeed release and remove lactate faster than adults? Applied Physiology, Nutrition, and Metabolism. 2015; 40(6): 634-635. Doi: https://doi.org/10.1139/apnm-2015-0092.

23. Engel FA, Sperlich B, Stockinger C, Härtel S, Bös,ac K, Holmbergd HC. The kinetics of blood lactate in boys during and following a single and repeated all-out sprints of cycling are different than in men. Applied Physiology, Nutrition, and Metabolism. 2015; 40(6): 623-631, https://doi.org/10.1139/apnm-2014-0370.

24. Beneke R, Leithäuser RM. Maximal Lactate Steady State's Dependence on Cycling Cadence. 2017; 12(3) 304-309. Doi: https://doi.org/10.1123/ijspp.2015-0573.

25. Rodrigues VD, Silva AG, Ávilla WR. Resposta do exercício físico nos níveis do hormônio cortisol: uma breve introdução. Revista Digital - Buenos Aires. 2008;13(120).

26. Vingren JL, Budnar RGJr, Amy L. McKenzie AA, Duplanty HYL, Show all DEL. The acute testosterone, growth hormone, cortisol and interleukin-6 response to $164-\mathrm{km}$ road cycling in a hot environment. J SPORT SCI. 2015; 34(8):694-699.

27. Cook CJ, Crewther BT, Kilduff LP. Are free testosterone and cortisol concentrations associated with training motivation in elite male athletes? Psychology of Sport and Exercise. 2013;14(6):882-5.

28. Thomas NE, Leyshon A, Hughes MG, Davies B, Graham M, Baker JS. The effect of anaerobic exercise on salivary cortisol, testosterone and immunoglobulin (A) in boys aged 15-16 years. European journal of applied physiology. 2009;107(4):455-61. 